\title{
Mendelian Randomization Analysis of Circulating Adipokines and C-reactive Protein on Breast Cancer Risk
}

Robinson $\mathrm{T}^{1}$, Martin $\mathrm{RM}^{1,2,3}$, Yarmolinsky $\mathbf{J}^{1,2}$

${ }^{1}$ Population Health Sciences, Bristol Medical School, University of Bristol, Bristol, UK

${ }^{2}$ MRC Integrative Epidemiology Unit, Population Health Sciences, Bristol Medical School, University of Bristol, Bristol, UK

${ }^{3}$ University Hospitals Bristol NHS Foundation Trust National Institute for Health Research Bristol Biomedical Research Centre, University of Bristol, Bristol, UK

Corresponding author:

Timothy Robinson, BMBS, PhD, MRCP

Bristol Cancer Institute

Horfield Rd

Bristol, United Kingdom

BS2 8ED

Tim.robinson@bristol.ac.uk

Word count: 1020 


\begin{abstract}
Adipokines and C-reactive protein (CRP) have been proposed as molecular mediators linking adiposity to breast cancer (BCa). Mendelian randomization (MR) uses genetic variants as proxies for risk factors to strengthen causal inference in observational studies. We performed a MR analysis to evaluate the causal relevance of six circulating adipokines (adiponectin, hepatocyte growth factor, interleukin-6, leptin receptor, plasminogen activator inhibitor-1, resistin) and CRP in risk of overall and oestrogen receptor-stratified $\mathrm{BCa}$ in up to 122,977 cases and 105,974 controls. Genetic instruments were constructed from single-nucleotide polymorphisms robustly $\left(P<5 \times 10^{-8}\right)$ associated with risk factors in genome-wide association studies. In MR analyses, there was evidence for a causal effect of hepatocyte growth factor on ER- BCa (OR per SD increase:1.17, 95\% CI: 1.01-1.35; $P=0.035)$ but little evidence for effects of other adipokines or CRP on overall or oestrogen receptor-stratified $\mathrm{BCa}$.

Collectively, these findings do not support an important etiological role of various adipokines or $\mathrm{CRP}$ in $\mathrm{BCa}$ risk.
\end{abstract}


Elevated body mass index (BMI) is an important modifiable risk factor for breast cancer $(\mathrm{BCa})(1)$ and adipokines - cytokines and hormones released by adipose tissue- are potential molecular mediators linking excess adiposity to $\mathrm{BCa}(2-4)$. In vitro studies have demonstrated that two adipokines in particular - leptin and adiponectin - may have pro- and antiproliferative effects on $\mathrm{BCa}$ cells, respectively, (5) and meta-analyses of observational studies support their opposing roles in $\mathrm{BCa}$ risk $(6,7)$. Observational studies have linked other adipokines including hepatocyte growth factor (HGF), interleukin-6 (IL-6), plasminogen activator inhibitor-1 (PAI-1), and resistin to BCa, albeit less consistently(8-10). Prediagnostic C-reactive protein (CRP), a systemic marker of inflammation that is partially synthesized by adipose tissue(11), has also been associated with BCa risk in prospective observational studies(12). Collectively, these findings suggest that pharmacological targeting of adipokines or CRP could be an effective strategy for BCa prevention among overweight and/or obese women. However, the causal nature of these risk factors in BCa risk is unclear as conventional observational analyses are susceptible to residual confounding and reverse causation, which undermine causal inference $(13,14)$.

Mendelian randomization (MR) uses genetic variants as instruments ("proxies") for risk factors to generate more reliable evidence on the causal effects of these factors on disease outcomes $(15,16)$. The use of genetic variants as instruments minimises confounding and precludes reverse causation as germline genotype is largely independent of lifestyle and environmental factors and is fixed at conception. The power and precision of MR analysis can be increased by a "two-sample MR" framework in which summary genetic association data from independent samples representing genetic variant-exposure and genetic variantoutcome associations are synthesised in order to estimate causal effects(17). 
Given uncertainty surrounding the role of various adipokines and CRP in BCa aetiology, we performed two-sample MR analyses to evaluate the potential causal role of circulating adiponectin, HGF, IL-6, leptin receptor, PAI-1, resistin, and CRP in overall and oestrogen receptor (ER)-stratified BCa risk.

Summary genome-wide association study (GWAS) statistics were obtained from analyses on 122,977 BCa cases (with further sub-analyses of 69,501 ER-positive (ER+) and 21,468 ERnegative (ER-) BCa cases) and 105,974 controls of European ancestry from the Breast Cancer Association Consortium (BCAC)(18). BCAC samples have the relevant ethical approval and genotyping was performed as previously described(18, 19).

Genetic instruments to proxy HGF, IL-6, leptin receptor, and resistin were constructed by obtaining individual cis-acting single-nucleotide polymorphisms (SNPs) robustly associated with these markers $\left(P<5 \times 10^{-8}\right)$ in GWAS of individuals of European ancestry that were replicated in independent samples. Cis-variants (located $\leq 1 \mathrm{MB}$ of the transcription start site of the protein-coding gene) are more likely to have direct effects on protein levels than transvariants (>1MB of the transcription start site of the protein-coding gene), minimising horizontal pleiotropy (an instrument influencing an outcome through one or more biological pathways independent to that of the exposure), a violation of the exclusion restriction criterion(16). For risk factors with $\geq$ three independent $\left(r^{2}<0.01\right)$ cis- or trans-SNPs available as proxies (adiponectin, CRP, PAI-1), these SNPs were combined into multi-allelic instruments to increase the variance in the risk factor explained by the instrument. As sensitivity analyses for adiponectin, CRP, and PAI-1, causal estimates generated from multiallelic instruments were compared with those obtained from instruments consisting of weakly correlated $\left(r^{2}<0.15\right)$ cis-variants to investigate horizontal pleiotropy in primary multi-allelic models. 
$R^{2}$ and F-statistics were calculated to examine the strength of our instruments, using previously reported methods(20). For instruments constructed using individual cis-variants, causal estimates were generated using the Wald ratio and standard errors were approximated using the delta method. For instruments constructed using $\geq$ three independent variants, causal estimates were generated using inverse-variance weighted (IVW) random effects models to account for overdispersion in models (21). If underdispersion in a model was present, the residual standard error was set to 1 . Sensitivity analyses for analyses employing multi-allelic instruments using weakly correlated cis-variants were performed using random-effects IVW models with adjustment for correlations between variants(22).

For each risk factor, the number of SNPs included in the instrument and estimates of instrument strength $\left(R^{2}\right.$ and F-statistics) are presented in Table 1 with F-statistics ranging from 19.0-3872.7, suggesting that analyses were unlikely to suffer from weak instrument $\operatorname{bias}(23)$.

In MR analyses, there was little evidence to suggest causal effects for any of the adipokines or CRP in overall breast cancer (Table 2). In ER status-stratified analyses, there was evidence for an effect of HGF on ER- BCa risk (OR per SD increase:1.17,95\%CI:1.01$1.35 ; P=0.035)$. Findings for adiponectin, PAI-1, and CRP using cis-SNP instruments were consistent with those using multi-allelic instruments (Supplementary Table 1).

Contrary to some conventional observational studies $(6-9,12)$, our MR analyses using genetic variants as proxies found little evidence to support causal roles for various adipokines or CRP in BCa risk. Our data support a causal role of circulating HGF in ER- BCa risk that is consistent with in vitro studies suggesting a role of HGF in tumour cell proliferation, migration, and invasion $(24,25)$ and observational studies reporting a relationship of HGF levels with more advanced $\mathrm{BCa}$ staging and worse prognosis $(10,26,27)$. While this result 
could be compatible with chance given the number of statistical tests performed, the alignment of findings from laboratory, observational, and genetic studies suggests the potential aetiological role of HGF in ER- BCa development.

Strengths of this analysis include the use of a two-sample MR framework that enabled increased statistical power and precision by exploiting summary genetic data from several large GWAS. There are several limitations to these analyses. First, since analyses were performed using summary genetic data in aggregate, this precluded stratification according to menopausal status. Second, though attempts were made to circumvent potential violations of MR assumptions in our analyses through the use of cis-acting variants as primary instruments and in sensitivity analyses, we cannot rule out the possibility that false negative findings may have arisen through horizontally pleiotropic pathways biasing our findings toward the null. Lastly, we were unable to examine possible non-linear effects of adipokines or CRP on BCa risk.

Overall, our findings suggest that several adipokines and CRP are unlikely to causally influence BCa risk. The potential aetiological role of HGF in ER- BCa warrants further investigation as a pharmacological target for $\mathrm{BCa}$ prevention. 
bioRxiv preprint doi: https://doi.org/10.1101/720110; this version posted July 31, 2019. The copyright holder for this preprint (which was not certified by peer review) is the author/funder, who has granted bioRxiv a license to display the preprint in perpetuity. It is made available under aCC-BY 4.0 International license. 


\section{Funding:}

TR is supported by the National Institute for Health Research (NIHR) as an Academic Clinical Lecturer in Medical Oncology. JY is supported by a Cancer Research UK (C18281/A19169) programme grant (the Integrative Cancer Epidemiology Programme Cancer Research UK Research PhD studentships (C18281/A20988). JY and RMM are supported by a Cancer Research UK (C18281/A19169) programme grant (the Integrative Cancer Epidemiology Programme) and are part of the Medical Research Council Integrative Epidemiology Unit at the University of Bristol supported by the Medical Research Council (MC_UU_12013/1, MC_UU_12013/2, and MC_UU_12013/3) and the University of Bristol. RMM is also supported by the National Institute for Health Research (NIHR) Bristol Biomedical Research Centre which is funded by the National Institute for Health Research (NIHR) and is a partnership between University Hospitals Bristol NHS Foundation Trust and the University of Bristol. Department of Health and Social Care disclaimer: The views expressed are those of the author(s) and not necessarily those of the NHS, the NIHR or the Department of Health and Social Care 


\section{References:}

1. Bhaskaran K, Douglas I, Forbes H, dos-Santos-Silva I, Leon DA, Smeeth L. Bodymass index and risk of 22 specific cancers: a population-based cohort study of 5.24 million UK adults. Lancet. 2014;384(9945):755-65.

2. Ando S, Gelsomino L, Panza S, Giordano C, Bonofiglio D, Barone I, et al. Obesity, Leptin and Breast Cancer: Epidemiological Evidence and Proposed Mechanisms. Cancers (Basel). 2019;11(1).

3. Gunter MJ, Wang T, Cushman M, Xue X, Wassertheil-Smoller S, Strickler HD, et al. Circulating Adipokines and Inflammatory Markers and Postmenopausal Breast Cancer Risk. J Natl Cancer Inst. 2015;107(9).

4. Vona-Davis L, Rose DP. Adipokines as endocrine, paracrine, and autocrine factors in breast cancer risk and progression. Endocr Relat Cancer. 2007;14(2):189-206.

5. Grossmann ME, Ray A, Nkhata KJ, Malakhov DA, Rogozina OP, Dogan S, et al. Obesity and breast cancer: status of leptin and adiponectin in pathological processes. Cancer Metastasis Rev. 2010;29(4):641-53.

6. Macis D, Guerrieri-Gonzaga A, Gandini S. Circulating adiponectin and breast cancer risk: a systematic review and meta-analysis. Int J Epidemiol. 2014;43(4):1226-36.

7. Pan H, Deng LL, Cui JQ, Shi L, Yang YC, Luo JH, et al. Association between serum leptin levels and breast cancer risk: An updated systematic review and meta-analysis.

Medicine (Baltimore). 2018;97(27):e11345.

8. Gui Y, Pan Q, Chen X, Xu S, Luo X, Chen L. The association between obesity related adipokines and risk of breast cancer: a meta-analysis. Oncotarget. 2017;8(43):75389-99.

9. Iacoviello L, Agnoli C, De Curtis A, di Castelnuovo A, Giurdanella MC, Krogh V, et al. Type 1 plasminogen activator inhibitor as a common risk factor for cancer and ischaemic vascular disease: the EPICOR study. BMJ Open. 2013;3(11):e003725.

10. Toi M, Taniguchi T, Ueno T, Asano M, Funata N, Sekiguchi K, et al. Significance of circulating hepatocyte growth factor level as a prognostic indicator in primary breast cancer. Clin Cancer Res. 1998;4(3):659-64.

11. Timpson NJ, Nordestgaard BG, Harbord RM, Zacho J, Frayling TM, TybjaergHansen A, et al. C-reactive protein levels and body mass index: elucidating direction of causation through reciprocal Mendelian randomization. Int J Obes (Lond). 2011;35(2):300-8. 12. Chan DS, Bandera EV, Greenwood DC, Norat T. Circulating C-Reactive Protein and Breast Cancer Risk-Systematic Literature Review and Meta-analysis of Prospective Cohort Studies. Cancer Epidemiol Biomarkers Prev. 2015;24(10):1439-49.

13. Boyko EJ. Observational research--opportunities and limitations. J Diabetes Complications. 2013;27(6):642-8.

14. Lawlor DA, Harbord RM, Sterne JA, Timpson N, Davey Smith G. Mendelian randomization: using genes as instruments for making causal inferences in epidemiology. Stat Med. 2008;27(8):1133-63.

15. Davey Smith G, Ebrahim S. 'Mendelian randomization': can genetic epidemiology contribute to understanding environmental determinants of disease? Int J Epidemiol. 2003;32(1):1-22.

16. Yarmolinsky J, Wade KH, Richmond RC, Langdon RJ, Bull CJ, Tilling KM, et al. Causal Inference in Cancer Epidemiology: What Is the Role of Mendelian Randomization? Cancer Epidemiol Biomarkers Prev. 2018;27(9):995-1010.

17. Pierce BL, Burgess S. Efficient design for Mendelian randomization studies: subsample and 2-sample instrumental variable estimators. Am J Epidemiol. 2013;178(7):1177-84. 
18. Michailidou K, Lindstrom S, Dennis J, Beesley J, Hui S, Kar S, et al. Association analysis identifies 65 new breast cancer risk loci. Nature. 2017;551(7678):92-4.

19. Amos CI, Dennis J, Wang Z, Byun J, Schumacher FR, Gayther SA, et al. The OncoArray Consortium: A Network for Understanding the Genetic Architecture of Common Cancers. Cancer Epidemiol Biomarkers Prev. 2017;26(1):126-35.

20. Burgess S. Sample size and power calculations in Mendelian randomization with a single instrumental variable and a binary outcome. Int J Epidemiol. 2014;43(3):922-9.

21. Bowden J, Davey Smith G, Burgess S. Mendelian randomization with invalid instruments: effect estimation and bias detection through Egger regression. Int J Epidemiol. 2015;44(2):512-25.

22. Burgess S, Zuber V, Valdes-Marquez E, Sun BB, Hopewell JC. Mendelian randomization with fine-mapped genetic data: Choosing from large numbers of correlated instrumental variables. Genet Epidemiol. 2017;41(8):714-25.

23. Pierce BL, Ahsan H, Vanderweele TJ. Power and instrument strength requirements for Mendelian randomization studies using multiple genetic variants. Int J Epidemiol. 2011;40(3):740-52.

24. Nakamura T, Matsumoto K, Kiritoshi A, Tano Y, Nakamura T. Induction of hepatocyte growth factor in fibroblasts by tumor-derived factors affects invasive growth of tumor cells: in vitro analysis of tumor-stromal interactions. Cancer Res. 1997;57(15):330513.

25. Sattler M, Salgia R. The MET axis as a therapeutic target. Update Cancer Ther. 2009;3(3):109-18.

26. Sheen-Chen SM, Liu YW, Eng HL, Chou FF. Serum levels of hepatocyte growth factor in patients with breast cancer. Cancer Epidemiol Biomarkers Prev. 2005;14(3):715-7. 27. Yamashita J, Ogawa M, Yamashita S, Nomura K, Kuramoto M, Saishoji T, et al. Immunoreactive hepatocyte growth factor is a strong and independent predictor of recurrence and survival in human breast cancer. Cancer Res. 1994;54(7):1630-3. 
Table 1. Number of SNPs included in instrument, estimate of the proportion of variance in risk factor explained by the instrument $\left(R^{2}\right)$, and F-statistic for each instrument, across all adipokines and $C$-reactive protein

\begin{tabular}{|lccc|}
\hline Risk factor & $\begin{array}{c}\text { Number of SNPs } \\
\text { in instrument }\end{array}$ & $\boldsymbol{R}^{2}$ & F-statistic \\
& 8 & 0.016 & \\
\hline Adiponectin & 31 & 0.0069 & 33.0 \\
C-reactive protein & 1 & 0.012 & 40.1 \\
Hepatocyte growth factor & 3 & 0.01 & 301.9 \\
Interleukin-6 & 1 & 0.54 & 3872.7 \\
Leptin receptor & 3 & 0.0064 & 65.5 \\
PAI-1 & 1 & 0.015 & 19.0 \\
Resistin & & & \\
\hline
\end{tabular}

SNPs = single-nucleotide polymorphisms, $R^{2}=$ proportion of variance in risk factor explained by genetic instrument, PAI-1 = Plasminogen activator inhibitor-1 
Table 2. Effect estimates per unit increase in adipokines or C-reactive protein on overall and oestrogen-receptor stratified breast cancer risk

\begin{tabular}{|c|c|c|c|c|c|c|}
\hline \multirow[t]{2}{*}{ Risk factor } & \multicolumn{2}{|c|}{ Overall breast cancer } & \multicolumn{2}{|c|}{ ER+ breast cancer } & \multicolumn{2}{|c|}{ ER- breast cancer } \\
\hline & OR $(95 \% \mathrm{CI})$ & $P$ value & OR $(95 \% \mathrm{CI})$ & $P$ value & OR $(95 \% \mathrm{CI})$ & $P$ value \\
\hline Adiponectin & $1.06(0.94-1.21)$ & 0.34 & $0.98(0.84-1.14)$ & 0.80 & $1.19(0.95-1.50)$ & 0.14 \\
\hline C-reactive protein & $0.96(0.88-1.05)$ & 0.38 & $0.96(0.86-1.06)$ & 0.46 & $1.03(0.87-1.21)$ & 0.74 \\
\hline Hepatocyte growth factor & $1.01(0.93-1.10)$ & 0.77 & $1.01(0.92-1.11)$ & 0.86 & $1.17(1.01-1.35)$ & 0.035 \\
\hline Interleukin-6 & $1.09(0.96-1.25)$ & 0.18 & $1.12(0.96-1.31)$ & 0.14 & $1.00(0.79-1.27)$ & 0.99 \\
\hline Leptin receptor & $1.00(0.99-1.01)$ & 0.63 & $1.00(0.99-1.01)$ & 0.81 & $1.00(0.98-1.02)$ & 0.78 \\
\hline PAI-1 & $0.97(0.85-1.10)$ & 0.64 & $1.02(0.87-1.19)$ & 0.81 & $0.95(0.75-1.20)$ & 0.64 \\
\hline Resistin & $1.04(0.95-1.13)$ & 0.38 & $1.05(0.95-1.17)$ & 0.33 & $1.01(0.86-1.18)$ & 0.92 \\
\hline
\end{tabular}

$\mathrm{ER}+=$ Oestrogen receptor positive, ER- = Oestrogen receptor negative, $\mathrm{PAI}-1=$ Plasminogen activator inhibitor- $1, \mathrm{OR}=\mathrm{Odds}$ Ratio, $95 \% \mathrm{CI}=95 \%$

Confidence Interval. Causal estimates represent the effect of a one unit increase in: natural log-transformed adiponectin, C-reactive protein, interleukin-6, and plasminogen activator inhibitor-1, and standardized hepatocyte growth factor, leptin receptor, and resistin 
bioRxiv preprint doi: https://doi.org/10.1101/720110; this version posted July 31, 2019. The copyright holder for this preprint (which was not certified by peer review) is the author/funder, who has granted bioRxiv a license to display the preprint in perpetuity. It is made available under aCC-BY 4.0 International license. 